\section{Constipation and colonic perforation complicating calcium resonium therapy}

Sir,

Constipation is a well recognized complication of treatment with many different medicines. ${ }^{1}$ Significant side effects of such constipation, however, are not common. Pseudo-obstruction induced by an amitriptyline overdose has been associated with intestinal perforation. ${ }^{2}$ We have treated a patient who developed absolute constipation and colonic perforation complicating the use of calcium resonium therapy.

A 62 year old male was admitted to hospital for treatment of chronic renal and cardiac failure. Drug therapy on admission was nifedipine, colchicine, sulphinpyrazone, probenecid, bumetanide daily, inhaled ipratropium bromide and fenoterol hydrobromide and codydramol for joint pain. He was commenced on captopril to improve his cardiac failure. Unfortunately, his serum potassium increased to $7.1 \mathrm{mmol} / \mathrm{l}$. He was, therefore, commenced on calcium resonium $15 \mathrm{~g}$ (increased subsequently to $30 \mathrm{~g}$ ) thrice daily. The serum potassium fell to $4.9 \mathrm{mmol} / \mathrm{l}$ and the patient was discharged from hospital continuing on calcium resonium $15 \mathrm{~g}$ thrice daily. Prior to discharge he had complained of constipation. Glycerol suppositories and lactulose $15 \mathrm{ml}$ daily were prescribed as laxatives.

The patient was subsequently admitted with a 48 hour history of absolute constipation. Abdominal X-ray showed the descending colon to be loaded with faecal material. The constipation was presumed to be secondary to calcium resonium therapy. Conservative treatment was unsuccessful. At laparotomy he was found to have perforation of the sigmoid colon. A Hartmann's procedure was performed with removal of $2 \mathrm{~kg}$ of inspissated faeces including calcium resonium. Despite further treatment the patient subsequently died.

Faecal impaction of calcium resonium has previously been reported in children following rectal administration of the drug and gastric concretions have been reported in neonates after oral administration. ${ }^{3}$ Constipation may also occur and normally responds to treatment with aperients. This case, however, reflects the possible severity of such constipation although to the knowledge of the manufacturer a similar case has not been reported.

There is no defined limit of calcium resonium dose that may be used. In this patient the hyperkalaemia was resistant to the starting dose necessitating a dose increase.

There are two possible contributing factors to this patient developing colonic obstruction. The patient had a normal bowel habit despite the use of drugs including codydramol which are associated with constipation. It is possible, however, that the addition of calcium resonium to these drugs increased the risk of developing large bowel obstruction. The patient had also been recommended to limit the intake of oral fluids to about 1.3 litres daily in view of his cardiac and chronic renal failure. Such a fluid restriction may have limited his ability to clear the resonium from the bowel.

It is suggested, therefore, that therapy with calcium resonium should be monitored so that possible interactions with other constipating agents can be avoided. In addition, adequate patient fluid intake and limitation of dosage should be ensured.
E.J. Minford T. Hand* M.C. Jones Departments of Surgery, and Medicine and Therapeutics,

Foresterhill,

University of Aberdeen, Aberdeen $A B 92 Z D$ and ${ }^{*}$ Department of Surgery, Dr Gray's Hospital, Elgin, $U K$.

\section{References}

1. Anuras, S. \& Baker, C.R. The colon in the pseudo-obstructive syndrome. Clin Gastroenterol 1986, 15: 745-762.

2. McMahon, A.J. Amitriptyline overdose complicated by intestinal pseudo-obstruction and caecal perforation. Postgrad Med J 1989, 65: 948-949.

3. ABPI data sheet compendium 1990-1991. Calcium resonium side effects, p. 1706.

\section{Rapid pulmonary cavitation due to Mycobacterium tuberculosis and infection with human immunodeficiency virus (HIV 1)}

Sir,

Infection with Mycobacterium tuberculosis is increasingly recognized among patients infected with the human immunodeficiency virus (HIV). ${ }^{1}$ Cavitation of pulmonary lesions in patients with tuberculosis (TB) and HIV is considered to be rare $^{2}$ but we have recently observed a rapidly expanding pulmonary cavity in a patient with HIV infection and pulmonary TB.

A 29 year old Ugandan businessman was admitted for investigation of a persistent cough, weight loss and diarrhoea. A diagnosis of pulmonary TB had been made 9 months previously in Uganda and he was treated for 3 months with streptomycin and thiacetazone. Six months later he arrived in England and was investigated at another hospital for cough, weight loss and a pruritic rash. His chest X-ray showed diffuse interstitial shadowing in both mid and upper zones. An antibody test for HIV 1 was positive. Bronchoalveolar lavage showed no organisms other than Haemophilus influenzae. He received oral cotrimoxazole for 2 weeks and because of possible recurrence of TB he received rifampicin, isoniazid and pyrazinamide. Culture of lavage fluid was subsequently sterile and his antituberculous therapy was discontinued after 3 months.

On admission to this hospital he was complaining of a productive cough, diarrhoea and malaise. He was a thin man with no lymphadenopathy and a rash due to chronic tinea corporis. He was pyrexial with scattered expiratory wheezes throughout the chest. Investigations showed a haemoglobin of $9.8 \mathrm{~g} / \mathrm{dl}$, total white cell count $9.46 \times 10^{9} /$ l, CD4 count $800 \times 10^{6} / 1$, and platelets $113 / \mathrm{nl}$. Urea, electrolytes and liver function tests were normal apart from an albumin of $28 \mathrm{~g} / \mathrm{dl}$. Blood cultures, thick films for malarial parasites and stool samples for ova, cysts, parasites and other pathogens were negative. Chest X-ray 